\title{
Fourier Cosine Series Method for Solving the Generalized Elastic Thin-walled Column Buckling Problem for Dirichlet Boundary Conditions
}

\author{
Charles C. Ike ${ }^{1 *}$, Hyginus N. Onah ${ }^{2}$, Benjamin O. Mama² ${ }^{2}$ Clifford U. Nwoji², Edwin U. Ikwueze ${ }^{1}$ \\ ${ }^{1}$ Department of Civil Engineering, Faculty of Engineering, Enugu State University of Science and Technology, Enugu 400001, \\ Enugu State, Nigeria \\ ${ }^{2}$ Department of Civil Engineering, Faculty of Engineering, University of Nigeria, Nsukka, Enugu State, Nigeria
}

Corresponding Author Email: ikecc2007@yahoo.com

https://doi.org/10.18280/rcma.290301

Received: 4 March 2019

Accepted: 22 May 2019

Keywords:
Fourier cosine series method, generalized
elastic thin-walled column buckling
problem, characteristic buckling
equation, algebraic eigenvalue problem,
eigenvalue, modal displacement
functions, critical buckling load

\section{Keywords:} problem, characteristic buckling equation, algebraic eigenvalue problem, functions, critical buckling load

\begin{abstract}
The Fourier cosine series method was used to obtain solutions to the generalized elastic thin-walled column buckling problem for the case of Dirichlet end boundary conditions. The problem is a boundary value problem given by a set of three coupled ordinary differential equations with three unknown displacement functions, and subject to the Dirichlet end boundary conditions. By choosing the origin of the longitudinal coordinate at the middle of the column, the Fourier cosine series was found to be a suitable shape function for the problem and hence the three displacement modal functions were represented using Fourier cosine series, with unknown modal amplitudes. The Fourier cosine series representation of the unknown displacement modal functions simplified the problem to an algebraic eigenvalue problem given by a set of homogenous algebraic equations whose nontrivial solution yielded the characteristic buckling equation. The stability equation was obtained as a third-degree polynomial for the asymmetric cross-sectioned column. For asymmetric cross-sections, the buckling modes were obtained as coupled flexural-torsional modes. For bisymmetric cross-sections, the buckling modes were uncoupled and failure could be flexural or flexural-torsional. For monosymmetric cross-sections, one of the buckling modes is uncoupled while the others are coupled; and failure could be by either Euler bending or bending-torsional buckling. The solutions obtained in this work agree with results obtained by previous researchers.
\end{abstract}

\section{INTRODUCTION}

Elastic thin-walled columns with monosymmetric, bisymmetric or asymmetric cross-sections are used in various engineering applications. Their materials could be isotropic or anisotropic, homogeneous or heterogeneous [1-8]. They are used as beams, columns or beam-columns in building structures, machine parts, bridge structures, space, aeroplanes, aeronautics, boats and ship structures. Their thin-walled cross-sections make them prone to buckling failures under compressive loads [7, 9-12].

The generalized elastic thin-walled column buckling problem is represented mathematically as a boundary value problem described by a set of complex ordinary differential equations (ODEs) and end boundary conditions. In the most general case, the problem is complex and nonlinear requiring complicated mathematical tools and are intractable for many cases of end supports [7]. Euler [9] was one of the first scholars that studied the elastic buckling behaviour of thin columns. Euler developed from first principles the ordinary differential equation (ODE) for the stability of columns. He further solved the governing ordinary differential equation for various end support conditions, thus obtaining the buckling modes and buckling loads.

Saint Venant [10] presented his work on the torsional behaviours of structural beams and columns; and developed expressions for the torsional buckling shapes and torsional buckling loads for different end supports. Michell [11] and Prandtl [12] presented analytical formulations for the lateral buckling behaviors of beams, columns and beam-columns, and developed analytical results for the flexural-torsional buckling loads for beams, columns and beam-column elements. Timoshenko [2, 13-15] considering warping torsion, developed the system of differential equations describing elastic buckling problems of beams, columns and beam-columns.

Further developments and research on the system of differential equations for the bending-torisonal stability analysis of beams, thin walled columns and beam-columns were due to researches carried out by many scholars such as Wagner [16], Vlasov [17], Timoshenko and Gere [2], Alsayed [18], Zlatko [19], Trahair [20-21], Allen and Bulson [22], Chajes [23], Avcar [24], Wang et al. [25], Det [26], Nwakali [27], Howlett [28], Zhu [29], and Al-Sheik [30].

Ike et al. [4] employed the Galerkinvariational method to obtain solutions to the boundary value problem (BVP) of a generalised elastic thin-walled column with pinned ends. They studied the problem for axial compressive loads concentrically applied at the cross-section centroid and for which the bimoment vanishes. They concluded that Galerkin method reduced the BVP to a set of homogeneous algebraic equations for which the nontrivial solutions gave the characteristic buckling equation as a third-degree polynomial with roots (eigenvalues) used to find the buckling loads [7]. 
Mama et al. [6] obtained closed form solutions to the generalised elastic column buckling problem using the finite Fourier sine transformation method [7]. They applied the finite Fourier sine transformation to the governing set of linear ODEs and found that the BVP transformed to an algebraic eigenvalue problem given as a set of homogeneous algebraic equations [7]. They used the methods of algebra to obtain nontrivial solutions as the characteristic buckling equation, which upon expansion is a cubic polynomial with roots used to find the buckling loads, and the critical buckling load. Onah et al. [5] used the Fourier sine series method to obtain the eigenvalues and eigenfunctions on the BVP of the generalized elastic thin-walled column buckling problem for the case of pinned ends [7]. They represented the three unknown displacement modal functions using Fourier sine series, which was constructed to satisfy the end boundary conditions. They found that the BVP got transformed to a system of homogeneous algebraic equations, representing an eigenvalue problem [7]. Methods of algebra were used to obtain nontrivial solution as the characteristic buckling equation which upon expansion was a third-degree polynomial with roots that were used to find the buckling loads, and the critical buckling load.

Ike et al. [7] used the least squares weighted residual method (LSWRM) to obtain solutions to the generalised elastic thin-walled column buckling problem for the case of pinned ends. The method was used to formulate the BVP as a variational problem of finding displacement modal buckling functions that minimized the functional defined as the square of the weighted residual. The method was found to simplify the problem to an algebraic eigenvalue problem which was solved for nontrivial cases to obtain the characteristic stability equation as a cubic polynomial. The roots were used to find all the buckling loads and the critical buckling load.

The aim of the study is to use the Fourier cosine series method to obtain solutions to generalized elastic thin-walled column buckling problem for Dirichlet boundary conditions. Mathematically, the problem is described by a set of three coupled ordinary differential equations (ODEs) with three unknown displacement functions $u(x), v(x)$ and $\theta(x)$; where $u(x)$ is the displacement component in the $y$ direction, $v(x)$ is the displacement component in the $z$ direction, and $\theta$ is the rotation, $x$ is the longitudinal axis of the thin-walled column.

The specific objectives are:

(i) to determine the buckling and modal shape functions for the set of three coupled equations in terms of Fourier cosine series that satisfy the Dirichlet boundary conditions at the two ends.

(ii) to simplify the solution of the boundary value problem (BVP) by reduction to an algebraic eigenvalue problem represented by a set of homogeneous equations in the buckling mode amplitudes.

(iii) to solve the resulting eigenvalue problem, and hence determine the characteristic buckling equation.

(iv) to determine the critical buckling loads for two particular cases: columns with bisymmetric crosssections and monosymmetric cross-sections.

\section{THEORETICAL FRAMEWORK}

The study strictly considered an elastic thin-walled column of length $l$, and with a longitudinal coordinate axis defined by the $x$ coordinate. The cross-sectional plane is defined by the $y z$ coordinates. The three coupled ordinary differential equations that govern the problem when the axial compressive load $Q_{x}$ is centroidal, no moments are due to transverse loads, and there is no applied torque and the bimoment vanishes are given as follows $[1-7,15]$ :

$$
\begin{gathered}
E I_{z z} \frac{d^{4} u(x)}{d x^{4}}+Q_{x} \frac{d^{2} u(x)}{d x^{2}}+Q_{x} e_{z} \frac{d^{2} \theta(x)}{d x^{2}}=0 \\
E I_{y y} \frac{d^{4} v(x)}{d x^{4}}+Q_{x} \frac{d^{2} v(x)}{d x^{2}}-Q_{x} e_{y} \frac{d^{2} \theta(x)}{d x^{2}}=0 \\
E C_{w} \frac{d^{4} \theta(x)}{d x^{4}}-\left(G J-\frac{I_{E} Q_{x}}{A}\right) \frac{d^{2} \theta(x)}{d x^{2}} \\
+e_{z} Q_{x} \frac{d^{2} u(x)}{d x^{2}}-e_{y} Q_{x} \frac{d^{2} v(x)}{d x^{2}}=0
\end{gathered}
$$

where, $u(x), v(x)$ and $\theta(x)$ are the displacement functions, $x$ is the longitudinal coordinate axis. $E$ is the Young's modulus of elasticity of the material of the column material, $G$ is the shear modulus or modulus of rigidity, $C_{w}$ is the warping constant, $I_{z z}$ is the moment of inertia about the $z$ coordinate axis, $I_{y y}$ is the moment of inertia about the $y$ coordinate axis, $e_{z}, e_{y}$ are coordinates of the shear center, $Q_{x}$ is the axial compressive load, $I_{E}$ is the polar moment of inertia about the shear center, $A$ is the area of the cross-section, $J$ is the Saint Venant torsional stiffness of cross-section. $I_{E}$ is expressed as:

$$
I_{E}=I_{y y}+I_{z z}+\left(e_{y}^{2}+e_{z}^{2}\right) A
$$

\section{METHOD}

\subsection{Dirichlet boundary conditions and Fourier cosine series for the buckling mode functions}

The origin of coordinates is chosen at the center of the column of length $l$, and the Dirichlet boundary conditions for the thin-walled column with pinned ends are:

$$
\begin{gathered}
u(x=-l / 2)=u(x=l / 2)=0 \\
u^{\prime \prime}(x=-l / 2)=u^{\prime \prime}(x=l / 2)=0 \\
v(x=-l / 2)=v(x=l / 2)=0 \\
v^{\prime \prime}(x=-l / 2)=v^{\prime \prime}(x=l / 2)=0 \\
\theta(x=-l / 2)=\theta(x=l / 2)=0 \\
\theta^{\prime \prime}(x=-l / 2)=\theta^{\prime \prime}(x=l / 2)=0
\end{gathered}
$$

where, the primes denote differentiation with respect to $x$.

Suitable Fourier cosine series that satisfy all the Dirichlet boundary conditions and can be used to represent the displacement buckling modal functions are given as: 


$$
\begin{aligned}
& u(x)=\sum_{n=1}^{\infty} u_{n} \cos \frac{n \pi x}{l} \\
& v(x)=\sum_{n=1}^{\infty} v_{n} \cos \frac{n \pi x}{l} \\
& \theta(x)=\sum_{n=1}^{\infty} \theta_{n} \cos \frac{n \pi x}{l}
\end{aligned}
$$

where, $u_{n}, v_{n}$ and $\theta_{n}$ are the amplitudes of the displacements for the $\mathrm{n} t h$ buckling mode.

\subsection{Application of the Fourier cosine series method}

Substitution of the displacement buckling modal equations - Equations (11-13) - into the governing elastic buckling equations - Equations (1-3), give:

$$
\begin{gathered}
E I_{z z} \frac{d^{4}}{d x^{4}} \sum_{n=1}^{\infty} u_{n} \cos \frac{n \pi x}{l}+Q_{x} \frac{d^{2}}{d x^{2}} \sum_{n=1}^{\infty} u_{n} \cos \frac{n \pi x}{l} \\
+Q_{x} e_{z} \frac{d^{2}}{d x^{2}} \sum_{n=1}^{\infty} \theta_{n} \cos \frac{n \pi x}{l}=0 \\
E I_{y y} \frac{d^{4}}{d x^{4}} \sum_{n=1}^{\infty} v_{n} \cos \frac{n \pi x}{l}+Q_{x} \frac{d^{2}}{d x^{2}} \sum_{n=1}^{\infty} v_{n} \cos \frac{n \pi x}{l} \\
-Q_{x} e_{y} \frac{d^{2}}{d x^{2}} \sum_{n=1}^{\infty} \theta_{n} \cos \frac{n \pi x}{l}=0 \\
E C_{w} \frac{d^{4}}{d x^{4}} \sum_{n=1}^{\infty} \theta_{n} \cos \frac{n \pi x}{l}-\left(G J-\frac{I_{E} Q_{x}}{A}\right) \frac{d^{2}}{d x^{2}} \\
\times \sum_{n=1}^{\infty} \theta_{n} \cos \frac{n \pi x}{l}+e_{z} Q_{x} \frac{d^{2}}{d x^{2}} \sum_{n=1}^{\infty} u_{n} \cos \frac{n \pi x}{l} \\
-e_{y} Q_{x} \frac{d^{2}}{d x^{2}} \sum_{n=1}^{\infty} v_{n} \cos \frac{n \pi x}{l}=0
\end{gathered}
$$

Simplifying,

$$
\begin{gathered}
\sum_{n=1}^{\infty}\left\{\left(E I_{z z}\left(\frac{n \pi}{l}\right)^{4}-Q_{x}\left(\frac{n \pi}{l}\right)^{2}\right) u_{n}\right. \\
\left.-Q_{x} e_{z}\left(\frac{n \pi}{l}\right)^{2} \theta_{n}\right\} \cos \frac{n \pi x}{l}=0 \\
\sum_{n=1}^{\infty}\left\{\left(E I_{y y}\left(\frac{n \pi}{l}\right)^{4}-Q_{x}\left(\frac{n \pi}{l}\right)^{2}\right) v_{n}\right. \\
\left.+Q_{x} e_{y}\left(\frac{n \pi}{l}\right)^{2} \theta_{n}\right\} \cos \frac{n \pi x}{l}=0
\end{gathered}
$$

$$
\begin{array}{r}
\sum_{n=1}^{\infty}\left\{\left(E C_{w}\left(\frac{n \pi}{l}\right)^{4}+\left(G J-\frac{I_{E} Q_{x}}{A}\right)\left(\frac{n \pi}{l}\right)^{2}\right) \theta_{n}\right. \\
\left.-Q_{x} e_{z}\left(\frac{n \pi}{l}\right)^{2} u_{n}+e_{y} Q_{x}\left(\frac{n \pi}{l}\right)^{2} v_{n}\right\} \cos \frac{n \pi x}{l}=0
\end{array}
$$

Further simplification by division by $(n \pi / l)^{2}$ yields:

$$
\begin{gathered}
\sum_{n=1}^{\infty}\left\{\left(E I_{z z}\left(\frac{n \pi}{l}\right)^{2}-Q_{x}\right) v_{n}-Q_{x} e_{z} \theta_{n}\right\} \cos \frac{n \pi x}{l}=0 \\
\sum_{n=1}^{\infty}\left\{\left(E I_{y y}\left(\frac{n \pi}{l}\right)^{2}-Q_{x}\right) v_{n}+Q_{x} e_{y} \theta_{n}\right\} \cos \frac{n \pi x}{l}=0 \\
\sum_{n=1}^{\infty}\left\{\left(E C_{w}\left(\frac{n \pi}{l}\right)^{2}+\left(G J-\frac{I_{E} Q_{x}}{A}\right)\right) \theta_{n}\right. \\
\left.-Q_{x} e_{z} u_{n}+e_{y} Q_{x} v_{n}\right\} \cos \frac{n \pi x}{l}=0
\end{gathered}
$$

\section{RESULTS}

\subsection{Reduction to algebraic eigenvalue problem}

For the $n$th buckling mode, the following system of homogeneous equations is obtained from the buckling modal amplitudes:

$$
\begin{array}{ccc}
\left(E I_{z z}\left(\frac{n \pi}{l}\right)^{2}-Q_{x}\right) & \begin{array}{c}
0 \\
\left.0 I_{y y}\left(\frac{n \pi}{l}\right)^{2}-Q_{x}\right)
\end{array} \\
-e_{z} Q_{x} & Q_{x} e_{y} Q_{x} \\
\times\left(\begin{array}{l}
u_{n} \\
v_{n} \\
\left.u_{w}\left(\frac{n \pi}{l}\right)^{2}+G J-\frac{I_{E} Q_{x}}{A}\right)
\end{array}\right) & \left(\begin{array}{l}
0 \\
0 \\
\theta_{n}
\end{array}\right)
\end{array}
$$

For nontrivial solutions of the set of homogeneous equations, the determinant of the coefficient matrix must vanish and this results in characteristic buckling (stability) equation as follows:

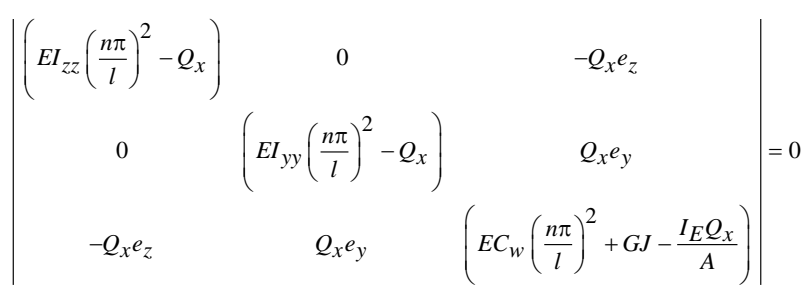


We define the buckling loads in pure flexure and torsion as follows:

$$
\begin{gathered}
P_{z z}^{E}=E I_{z z}\left(\frac{n \pi}{l}\right)^{2} \\
P_{y y}^{E}=E I_{y y}\left(\frac{n \pi}{l}\right)^{2} \\
P^{\phi}=\frac{A}{I_{E}}\left(E C_{w}\left(\frac{n \pi}{l}\right)^{2}+G J\right)
\end{gathered}
$$

Hence,

$$
E C_{w}\left(\frac{n \pi}{l}\right)^{2}+G J=\frac{P^{\phi} I_{E}}{A}
$$

where, $P_{z z}^{E}$ is the Euler flexural buckling load about the $z z$ axis, $P_{y y}^{E}$ is the Euler flexural buckling load about the $y y$ axis, and $P^{\phi}$ is the pure torsional buckling load.

The characteristic buckling equation is then given as:

$$
\left|\begin{array}{ccc}
\left(P_{z z}^{E}-Q_{x}\right) & 0 & -Q_{x} e_{z} \\
0 & \left(P_{y y}^{E}-Q_{x}\right) & Q_{x} e_{y} \\
-Q_{x} e_{z} & Q_{x} e_{y} & \left(\frac{P^{\phi} I_{E}}{A}-\frac{Q_{x} I_{E}}{A}\right)
\end{array}\right|=0
$$

Expansion of the characteristic buckling equation yields:

$$
\begin{array}{r}
\left(P_{z z}^{E}-Q_{x}\right)\left|\begin{array}{cc}
\left(P_{y y}^{E}-Q_{x}\right) & Q_{x} e_{y} \\
Q_{x} e_{y} & \frac{P^{\phi} I_{E}}{A}-\frac{Q_{x} I_{E}}{A}
\end{array}\right| \\
-Q_{x} e_{z}\left|\begin{array}{cc}
0 & \left(P_{y y}^{E}-Q_{x}\right) \\
-Q_{x} e_{z} & Q_{x} e_{y}
\end{array}\right|=0
\end{array}
$$

Expanding further,

$$
\begin{gathered}
\left(P_{z z}^{E}-Q_{x}\right)\left(\left(P_{y y}^{E}-Q_{x}\right)\left(P^{\phi}-Q_{x}\right) \frac{I_{E}}{A}-\left(Q_{x} e_{y}\right)^{2}\right) \\
-Q_{x} e_{z}\left(0+Q_{x} e_{z}\left(P_{y y}^{E}-Q_{x}\right)\right)=0
\end{gathered}
$$

Simplifying,

$$
\begin{gathered}
\left(P_{z z}^{E}-Q_{x}\right)\left(P_{y y}^{E}-Q_{x}\right)\left(P^{\phi}-Q_{x}\right) \frac{I_{E}}{A} \\
-\left(P_{z z}^{E}-Q_{x}\right)\left(Q_{x} e_{y}\right)^{2}-\left(Q_{x} e_{z}\right)^{2}\left(P_{y y}^{E}-Q_{x}\right)=0
\end{gathered}
$$

Multiplying by $\left(A / I_{E}\right)$, gives:

$$
\begin{gathered}
\left(P_{z z}^{E}-Q_{x}\right)\left(P_{y y}^{E}-Q_{x}\right)\left(P^{\phi}-Q_{x}\right)-\frac{A}{I_{E}}\left(P_{z z}^{E}-Q_{x}\right)\left(Q_{x} e_{y}\right)^{2} \\
-\frac{A}{I_{E}}\left(Q_{x} e_{z}\right)^{2}\left(P_{y y}^{E}-Q_{x}\right)=0 \\
\text { But } r_{0}^{2}=e_{y}^{2}+e_{z}^{2}+\left(\frac{I_{x x}+I_{z z}}{A}\right)
\end{gathered}
$$

where, $r_{0}$ is the radius of gyration.

Hence from Equation (4), we have

$$
\begin{aligned}
& \frac{I_{E}}{A}=\frac{I_{x x}+I_{y y}+\left(e_{y}^{2}+e_{z}^{2}\right) A}{A} \\
& =\frac{I_{x x}+I_{y y}}{A}+\left(e_{y}^{2}+e_{z}^{2}\right)=r_{0}^{2}
\end{aligned}
$$

Hence, the characteristic buckling equation becomes:

$$
\begin{gathered}
\left(P_{z z}^{E}-Q_{x}\right)\left(P_{y y}^{E}-Q_{x}\right)\left(P^{\phi}-Q_{x}\right) \\
-\left(P_{z z}^{E}-Q_{x}\right) \frac{\left(Q_{x} e_{y}\right)^{2}}{r_{0}^{2}}-\frac{\left(Q_{x} e_{z}\right)^{2}}{r_{0}^{2}}\left(P_{y y}^{E}-Q_{x}\right)=0
\end{gathered}
$$

Simplifying, we obtain:

$$
\begin{gathered}
\left(P_{z z}^{E}-Q_{x}\right)\left[\left(P_{y y}^{E}-Q_{x}\right)\left(P^{\phi}-Q_{x}\right)-\left(\frac{Q_{x} e_{y}}{r_{0}}\right)^{2}\right] \\
-\left(\frac{Q_{x} e_{z}}{r_{0}}\right)^{2}\left(P_{y y}^{E}-Q_{x}\right)=0
\end{gathered}
$$

Alternatively,

$$
\begin{gathered}
\left(P_{y y}^{E}-Q_{x}\right)\left[\left(P_{z z}^{E}-Q_{x}\right)\left(P^{\phi}-Q_{x}\right)-Q_{x}^{2} \frac{e_{z}^{2}}{r_{0}^{2}}\right] \\
-\left(P_{y y}^{E}-Q_{x}\right) Q_{x}^{2} \frac{e_{y}^{2}}{r_{0}^{2}}=0
\end{gathered}
$$

Eq. (36) is the characteristic stability equation for the determination of the buckling loads of a generalised elastic thin-walled column with an asymmetrical cross-section and for Dirichlet boundary conditions at the ends. The stability equation is a third degree polynomial in $Q_{x}$, and it has three zeros. The three zeros of the characteristic stability equations, called the three eigenvalues, are found by solving the characteristic stability equation using the numerical and analytical methods for solving polynomials. The least value of the three eigenvalues called the critical buckling load will govern the stability behaviour.

\subsection{Special cases of the generalised elastic thin-walled column buckling problem}

Two special cases, considered simplifications of the problem namely: (i) columns with bisymmetric cross- 
sections about the $y$ and $z$ coordinate axes, (ii) columns with monosymmetric cross-sections about one coordinate axis either the $y$ or the $z$ axis.

(i) Bisymmetric cross-sections

For bisymmetric cross-sections $e_{z}=e_{y}=0$. The characteristic buckling equation simplifies to:

$$
\left|\begin{array}{ccc}
\left(P_{z z}^{E}-Q_{x}\right) & 0 & 0 \\
0 & \left(P_{y y}^{E}-Q_{x}\right) & 0 \\
0 & 0 & \left(P^{\phi}-Q_{x}\right) \frac{I_{E}}{A}
\end{array}\right|=0
$$

Expansion yields the characteristic stability equation as the third degree polynomial in $Q_{x}$ :

$$
\left(P_{z z}^{E}-Q_{x}\right)\left(P_{y y}^{E}-Q_{x}\right)\left(P^{\phi}-Q_{x}\right) \frac{I_{E}}{A}=0
$$

The eigenvalues are found as:

$$
\begin{gathered}
Q_{x}=P_{z z}^{E}=E I_{z z}\left(\frac{n \pi}{l}\right)^{2} \\
Q_{x}=P_{y y}^{E}=E I_{y y}\left(\frac{n \pi}{l}\right)^{2} \\
Q_{x}=P^{\phi}=\frac{A}{I_{E}}\left(E C_{w}\left(\frac{n \pi}{l}\right)^{2}+G J\right)
\end{gathered}
$$

The buckling modes are decoupled in this case, since the governing stability equations are decoupled.

(ii) Monosymmetric cross-sections

If the axis of symmetry is the $z z$ axis, then $e_{y}=0, e_{z} \neq 0$, and the equation simplifies to:

$$
\left|\begin{array}{ccc}
\left(P_{z z}^{E}-Q_{x}\right) & 0 & -Q_{x} e_{z} \\
0 & \left(P_{y y}^{E}-Q_{x}\right) & 0 \\
-Q_{x} e_{z} & 0 & \frac{I_{E}}{A}\left(P^{\phi}-Q_{x}\right)
\end{array}\right|=0
$$

Expansion yields:

$$
\left(P_{y y}^{E}-Q_{x}\right)\left[\left(P_{z z}^{E}-Q_{x}\right)\left(P^{\phi}-Q_{x}\right)-Q_{x}^{2} \frac{e_{z}^{2}}{r_{0}^{2}}\right]=0
$$

Hence there are two possibilities for the roots, namely:

$$
P_{y y}^{E}-Q_{x}=0
$$

Or

$$
\left(P_{z z}^{E}-Q_{x}\right)\left(P^{\phi}-Q_{x}\right)-Q_{x}^{2}\left(\frac{e_{z}}{r_{0}}\right)^{2}=0
$$

\section{DISCUSSION}

The Fourier cosine series method has been successfully used in this research to obtain solutions to the generalized elastic thin-walled column buckling problem for the case of Dirichlet boundary conditions. The problem considered in this work is described using a set of three coupled ordinary linear differential equations (ODEs) given by Equations (1-3) in terms of the three displacement modal functions $u(x), v(x)$ and $\theta(x)$. For Dirichlet boundary conditions given by Equations (5-10) for the origin taken at the middle of the column, the three unknown displacement modal buckling functions that satisfied the boundary conditions were given in terms of Fourier cosine series of infinite terms as Equations (11-13) where the displacement amplitudes $u_{n}, v_{n}$, and $\theta_{n}$ for the $n$th buckling mode were unknown.

By the Fourier cosine series method, the substitution of the displacement modal buckling functions into the governing system of ODEs simplified the boundary value problem (BVP) to an algebraic problem given by the set of algebraic homogeneous equations in Equation (23). Following the methods for solving algebraic eigenvalue problems, the requirement for nontrivial solution of the homogeneous algebraic equations given by the vanishing of the determinant of the coefficient matrix was used to find the characteristic buckling equation as Equation (24). This was further expressed in terms of the flexural buckling load expressions about the two axes of the cross-section $\left(P_{y y}^{E}, P_{z z}^{E}\right)$ and pure torsional buckling load expression $\left(P^{\phi}\right)$ as Equation (29). Expansion of the characteristic buckling equation presented in determinantal form and algebraic simplifications resulted in the characteristic stability equation for the general asymmetric cross-section as the third degree polynomial in terms of $Q_{x}$ expressed as Equation (36). The characteristic buckling equation for the elastic column buckling problem for Dirichlet end boundary conditions and for asymmetric cross-section was further simplified to obtain Equations (37) or (38). The solution of the characteristic buckling equation using analytical and numerical methods for solving nonlinear and transcendental equations enable the determination of the three eigenvalues, which are used to find the three possible buckling loads. The minimum buckling load is the critical buckling load which governs the stability behaviour of the thin-walled column and determines the buckling mode as flexural or torsional or flexural-torsional. Two simplifications of the generalized problem were further considered monosymmetric and bisymmetric cross-sectioned thin-walled columns. For bisymmetric columns, the governing equations were found to be uncoupled, resulting to characteristic buckling equations that are uncoupled and given as the matrix Equation (39) with vanishing non diagonal terms. The solution of the uncoupled characteristic equation gave roots (eigenvalues) and buckling modes that are uncoupled. For columns with bisymmetric cross-sections, the buckling failure could proceed by either pure Euler flexural buckling about the $y y$ axis or the $z z$ axis or by pure torsional buckling. The least value of the three eigenvalues give the critical buckling load and would govern the failure of the elastic column with bisymmetrical cross-sections.

For thin-walled columns with monosymmetric crosssection where the axis of symmetry is the $z z$ axis, the 
characteristic stability equation was found as Equation (44). Expansion of the determinant yielded a third degree polynomial in $Q_{x}$ given as Equation (45). The solution gave two possibilities for the buckling failure. The first possibility is a pure flexural buckling given by Equation (46). The second possibility is represented by the quadratic equation (second degree polynomial) in $Q_{x}$, yielding two flexuraltorsional buckling loads. The smallest buckling load (eigenvalue) is the critical buckling load and would govern the buckling failure. The solutions for the buckling loads agree with previous solutions given by Det [26], Wang et al [25], Mama et al. [6] who used the finite Fourier sine transformation method, Onah et al [5] who used the Fourier sine series method, Ike et al [7] who applied the least squares weighted residual method.

\section{CONCLUSION}

The conclusions of the work are as follows:

(i) the Fourier cosine series method is an effective analytical technique for obtaining solutions to the boundary value problem (BVP) of elastic buckling of thin-walled column for Dirichlet end boundary conditions.

(ii) the method transformed the BVP of a set of coupled ordinary differential equations (ODEs) to an algebraic eigenvalue problem given by a set of homogeneous algebraic equations in terms of the amplitude of the modal displacements.

(iii) the characteristic buckling equation was obtained as a third degree polynomial in terms of $Q_{x}$; and the three zeros of the polynomial are the eigenvalues which are the three buckling loads.

(iv) for generalised elastic thin-walled column buckling problems with bisymmetric cross-sections, the governing stability equations are uncoupled, resulting in a system of homogeneous algebraic equations that are uncoupled, and characteristic stability equations with decoupled buckling loads and decoupled buckling modes.

(v) for generalised elastic thin-walled column buckling problems that have monosymmetric cross-sections with $z z$ axis as the axis of symmetry, the Euler bending buckling mode about the $y$ axis is decoupled while the Euler bending buckling mode about $z$ axis is coupled with torsional buckling.

(vi) for generalised elastic thin-walled column buckling problems with asymmetric cross-sections, the governing stability equations are a set of three coupled ordinary differential equations in the three displacement functions. The characteristic buckling equation is coupled. The two Euler flexural buckling modes about the two axes of the cross-section interact with the torsional buckling mode.

(vii) the expressions obtained in this study for the characteristic buckling equations and their eigenvalues were exact solutions for the Dirichlet boundary conditions considered since the Fourier cosine series which were the exact displacement modal shape functions that satisfied all the natural and essential boundary conditions at the ends were employed.

\section{REFERENCES}

[1] Torsion in Structural Design. Available: http://people.virginia.edu/ttb/torsion.pdf

[2] Timoshenko, S.P., Gere, J.M. (1961). Theory of Elastic Stability. New York: McGraw Hill Koga Kusha Ltd.

[3] Ike, C.C. (2018). Energy formulation for the flexuraltorsional buckling of thin-walled column with open cross-section. Mathematical Modelling of Engineering Problems (MMEP), 5(2): 58-66. https://doi.org/10.18280/mmep.050202

[4] Ike, C.C., Nwoji, C.U., Ikwueze, E.U., Ofondu, I.O. (2017). Solution of the generalized elastic column buckling problem by the Galerkin variational method. International Journal for Research in Applied Science and Engineering Technology (IJRASET), 5(1): 468-475.

[5] Onah, H.N., Ike, C.C., Nwoji, C.U. (2017). Flexuraltorsional buckling analysis of thin walled columns using the Fourier series method. International Journal of Advanced Engineering Research and Science (IJAERS), 4(3): 292-298. https://dx.doi.org/10.22161/ijaers.4.3.45

[6] Mama, B.O., Ike, C.C., Nwoji, C.U., Onah, H.N. (2017). Application of the finite Fourier sine transform method for the flexural-torsional buckling analysis of thinwalled columns. IOSR Journal of Mechanical and Civil Engineering (IOSR JMCE), 14(2): 51-60. https://dx.doi.org/10.9790/1684.1402015160

[7] Ike, C.C., Nwoji, C.U., Mama, B.O., Onah, H.N. (2019). Least squares weighted residual method for solving the generalized elastic column buckling problem. TecnicaItaliana - Italian Journal of Engineering Science, 63(1): 78-85. https://doi.org/10.18280/ti-ijes63.01.11

[8] Riley, C.E. (2003). Elastic buckling loads of slender columns with variable cross-section by the Newmark method. MSc Thesis. Department of Civil Engineering, Colorado State University.

[9] Euler, I. (1982). Sur la force des colonnes. Memoires de l'academie des sciences de Berlin, 1757, 13: 252-282 in Opera Omnia set 2 Vol 17, 1982, pp. 89-118.

[10] Saint Venant, A.J.C.B. (1855). Memoire sur la torsion des Prismes Mem Divers Savants, 14: 233-560.

[11] Michell, A.G.M. (1899). Elastic stability of long beams under transverse forces. Philos Mag., 48, 5th Series, 1899, pp. 298-309.

[12] Prandtl, L. (1899). Kipperscheinungen Dissertation des Universitat Munchen.

[13] Timoshenko, S.P. (1905). On the stability in pure bending of a T beam. Bull Pol. Ins St Petersburg, pp. 45.

[14] Timoshenko, S.P. (1936). Theory of Elastic Stability. New York: McGraw Hill.

[15] Timoshenko, S.P. (1945). Theory of bending torsion and buckling of thin walled members of open crosssection. Journal of the Franklin Institute, 239(5): 343361. https://doi.org/10.1016/0016-0032(45)90013-5

[16] Wagner, H. (1936). Torsion and Buckling of Open Sections T.M. No 807 US National Advisory Committee for Aeronautics.

[17] Vlasov, V.Z. (1961). Thin walled elastic beams. English translation, National Science Foundation, Washington DC London Oldbourne Press.

[18] Alsayed, S.H. (1987). Inelastic behaviour of single angle columns. PhD Thesis. The University of Arizona, University Microfilms 
http//hdl.handlev.net/10150/184041.

[19] Zlatko, T.Z. (2012). Stress and strain deflection of an open profile thin walled beam at constrained torsion by boundary element method. Journal of Theoretical and Applied Mechanics Sophia, 42: 43-54.

[20] Trahair, N.S. (1993). Flexural-Torsional Buckling of Structures. CRC Press Ann Arbor.

[21] Trahair, N.S. (2016). Torsion equations for lateral buckling. Research report R 964 July 2016, School of Engineering, The University of Sydney.

[22] Allen, H.G., Bulson P.S. (1980). Background to Buckling. London: McGraw Hill Book Company.

[23] Chajes, A. (1974). Principles of Structural Stability Theory. New Jersey: Prentice Hall.

[24] Avcar, M. (2014). Elastic buckling of steel columns under axial compression. American Journal of Civil Engineering, 2(3):

102-108. https://doi.org/10.11648/j.ajce.20140203.17

[25] Wang, C.M., Wang, C.Y., Reddy, J.N. (2005). Exact solution for buckling of structural members. CRC series in Computational Mechanics and Applied Mechanics, CRC Press USA.

[26] Det, N.V. (2004). Buckling strength analysis of bars and frames, and spherical shells classification notes No 30, 1, Norway, April 2004.

[27] Nwakali, J.A. (1990). The collapse behaviour of double layer space trusses incorporating eccentrically loaded tee-section members. PhD Thesis, Department of Civil Engineering, University of Surrey.

[28] Howlett, J.N. (1972). An investigation into the structural behaviour of thin walled aluminium alloy welded battered struts. MSc Thesis, University of Durham. http//ethesis.dur.ac.uk/10293.

[29] Zhu, S. (2009). Elastic flexural torsional buckling analysis of doubly symmetrical web tapered beams. MSc Thesis, University of Pittsburg, pp. 208.

[30] Al-Sheikh, A.M.S. (1985). Behavior of thin walled structures under combined loads. $\mathrm{PhD}$ Thesis. Loughborough University of Technology. 